\title{
Robust optimization methodologies for water supply systems design
}

\author{
J. Marques ${ }^{1}$, M. C. Cunha ${ }^{1}$, J. Sousa ${ }^{2}$, and D. Savić ${ }^{3}$ \\ ${ }^{1}$ Departamento de Engenharia Civil, Faculdade de Ciências e Tecnologia da Universidade de Coimbra, Portugal \\ ${ }^{2}$ Departamento de Engenharia Civil, Instituto Superior de Engenharia de Coimbra, Portugal \\ ${ }^{3}$ Centre for Water Systems, School of Engineering, Computing and Mathematics, University of Exeter, UK
}

Correspondence to: J. Marques (jmarques@ dec.uc.pt)

Received: 26 March 2012 - Published in Drink. Water Eng. Sci. Discuss.: 18 April 2012

Revised: 9 July 2012 - Accepted: 9 August 2012 - Published: 15 August 2012

\begin{abstract}
Water supply systems (WSSs) are vital infrastructures for the well-being of people today. To achieve good customer satisfaction the water supply service must always be able to meet people's needs, in terms of both quantity and quality. But unpredictable extreme conditions can cause severe damage to WSSs and lead to poorer levels of service or even to their failure. Operators dealing with a system's day-to-day operation know that events like burst water mains can compromise the functioning of all or part of a system. To increase a system's reliability, therefore, designs should take into account operating conditions other than normal ones. Recent approaches based on robust optimization can be used to solve optimization problems which involve uncertainty and can find designs which are able to cope with a range of operating conditions. This paper presents a robust optimization model for the optimal design of water supply systems operating under different circumstances. The model presented here uses a hydraulic simulator linked to an optimizer based on a simulated annealing heuristic. The results show that robustness can be included in several ways for varying levels reliability and that it leads to more reliable designs for only small cost increases.
\end{abstract}

\section{Introduction}

Modern societies are sustained by a number of vital networks. Energy, telecommunications, transport, water and sanitary infrastructures are responsible for a good quality of life. A disruption in the water supply can cause enormous trouble, which means that the systems have to be designed to deliver a constant supply of clean, safe drinking water, even in adverse circumstances. Every WSS will certainly have to contend with some burst pipes and abnormal demands, such as from firefighting. These events can have a minor or major impact on the operation of the WSSs and it is very important to maintain the supply and quality of water. According to DIEDE and AIDIS (2008), studies of hundreds of disasters worldwide clearly indicate that continuity of drinking water and sanitation services is critical in post-disaster conditions, since they are essential to rapid social and productive recovery. Water can still be provided, even in adverse situations, if a proactive attitude is taken towards risk from the design phase until the end of the system's life span. However, it must be pointed out that if all the possible threats and vulnerabilities could be taken into account the cost would be prohibitive. Hence, decision makers must establish how much they are willing to pay to reduce risk. As a WSS is a costly infrastructure its design and operation should be supported by optimization tools. Stochastic optimization and robust optimization (RO) appear to be promising techniques to solve these problems: the review by Mulvey et al. (1995) examines this area and describes some practical applications. RO has already been applied to WSS: Babayan et al. (2007), Jeong et al. (2006), Cunha and Sousa (2010), Carr et al. (2006) and Giustolisi et al. (2009) present a number of robust optimization models.

The model proposed by Cunha and Sousa (2009) for the robust design of water distribution networks includes multiple scenarios in the optimization model. These scenarios include the traditional peak discharge design and some 
abnormal working conditions like firefighting flows and pipe breaks. This approach also considers two levels of pressure: the desired pressure (minimum pressure to meet water demand) and the admissible pressure (minimum pressure allowed for the abnormal conditions scenarios). The pressure for the peak discharge design scenario is always higher than the desired pressure and so the network must be designed to meet the water demand under normal working conditions. The pressure for the abnormal scenarios is allowed to take lower values, although they are always higher than the admissible pressure. However, if the pressure is lower than the desired pressure then part of the water demand will not be met and the objective function is penalized.

The solutions obtained with this method showed that a robust design, a design that will meet all the desired pressure requirements even under abnormal working conditions, can be considerably more expensive than the traditional design solution (peak discharge design). As the case study used in Cunha and Sousa (2009) was a gravity fed water distribution network, the pipe diameters had to be increased to meet the pressure conditions in all scenarios, and consequently this added to the cost. For example, if the water demand is to be fully met during a pipe bursts the flow needs alternative paths to reach the demand nodes downstream of the break, and those paths must have enough capacity to carry a discharge that is higher than usual. As the pipe cost increases significantly with the diameter, this additional capacity is quite expensive. It must also be pointed out that larger diameters lead to low velocities and high water residence times, neither of which are desirable in terms of water quality and safety.

This paper proposes a different approach. As larger pipe diameters significantly increase the cost and lead to low velocities, it might be possible to cope with abnormal working conditions, which occur sporadically and last a short time, by adding a pumping station to be used like a contingency infrastructure. The strategy of this work involves a gravity fed network design to cater at least for normal working conditions (peak design flow) and a pumping station to add energy to cope with abnormal working conditions. The pumping station will only be planned to operate under abnormal working conditions, so the energy consumption can be neglected. It was also taken that the pressure under abnormal working conditions could be higher than under normal working conditions, but never above a maximum pressure constraint introduced in the optimization model. This will limit the elevation of the pumping station in abnormal conditions only to safe levels of operation.

With this contingency infrastructure, the network does not need to be overdesigned to attain the desired robustness, and this reduces the complications that can arise from low velocity problems. It can also be viewed as another way to increase robustness in an existing WSS where solutions such as increasing the pipe diameters may be hard to implement in an urban environment.
The optimization model is presented next, in Sect. 2, then the model is tested on 2 case studies in Sect. 3 and the results and comparisons are presented in Sect. 4. Finally, the conclusions are set out in Sect. 5.

\section{Robust model}

The model proposed here is based on the work by Cunha and Sousa (2009) and is used for the robust design of WSSs exposed to different operating scenarios. But a new approach to achieving the desired robustness is considered now, one which uses a pumping station instead of increasing the pipe diameters. The goal of the model is to find designs that will perform well even under abnormal conditions (pipe breaks or firefighting). The optimization model is solved by the simulated annealing algorithm proposed in Aarts and Korst (1989), used by Cunha and Sousa (1999) and Cunha and Sousa (2001) and adapted for this work. The model is linked to a hydraulic simulator that verifies the hydraulic constraints. An hydraulic simulator based on a pressure driven approach is used to verify the hydraulic constraints. Considering the sum of probabilities of all the scenarios to be 1, the objective function is formulated in Eq. (1):

$$
\begin{aligned}
\text { Min } & \sum_{i=1}^{\text {NPI }} C \operatorname{pipe}_{i}\left(D_{i}\right) L_{i}+\sum_{j=1}^{\mathrm{NPU}}\left(\mathrm{CCps}_{j}+\mathrm{CEps}_{j}\right) \\
& +\sum_{s=1}^{\mathrm{NS}} \operatorname{prob}_{s}\left[C \text { penp } \cdot \sum_{n=1}^{\mathrm{NN}} \max \left\{0 ;\left(P \mathrm{MINdes}_{s}-P_{n, s}\right)\right\}^{2}\right. \\
& \left.+C \text { pend } \cdot \sum_{n=1}^{\mathrm{NN}} \max \left\{0 ;\left(Q D_{n, s}-Q C_{n, s}\right)\right\}^{2}\right]
\end{aligned}
$$

Where $\mathrm{CCps}_{j}$ is the construction cost and $\mathrm{CEps}_{j}$ the equipment cost in $€$ of the pumping station (PS) $j$ :

$$
\begin{aligned}
& \mathrm{CCps}_{j}=39904+374 \times Q \mathrm{ps}_{j}+0.15 \times Q \mathrm{ps}_{j} \\
& \quad \times H \mathrm{ps}_{j} \forall j \in \mathrm{NPU}, \\
& \mathrm{CEps}_{j}=1317 \times Q \mathrm{ps}_{j}^{0.769} \times H \mathrm{ps}_{j}^{0.184}+2092 \\
& \quad \times\left(Q \mathrm{ps}_{j} \times H \mathrm{ps}_{j}\right)^{0.466} \forall j \in \mathrm{NPU} .
\end{aligned}
$$

The objective function Eq. (1) includes the following costs: cost of the pipes and cost of the pumping stations (construction and equipment). But it also includes a penalty function for those solutions that do not meet the minimum desired pressure and demands: the sum of the quadratic violations of pressures and demands multiplied by penalty coefficients and weighted by the probability of occurrence of each scenario.

The model includes a different set of constraints. Equation (4) is used to verify the nodal continuity equations; Eq. (5) is used to compute the head loss of the pipes; Eq. (6) is used to limit the pressure of the nodes and Eq. (7) is used to guarantee a minimum diameter for the pipes.

$\sum_{i=1}^{\mathrm{NPI}} I_{n, i} Q_{i, s}=\mathrm{QC}_{n, s} \forall n \in \mathrm{NN} ; \forall s \in \mathrm{NS}$, 
$\Delta H_{i, s}=K_{i} Q_{i, s}^{\alpha} \forall i \in \mathrm{NPI} ; \forall s \in \mathrm{NS}$,

$P \mathrm{MAX}_{n, s} \geq P_{n, s} \geq P \mathrm{MINadm}_{n, s} \forall n \in \mathrm{NN} ; \forall s \in \mathrm{NS}$,

$D_{i} \geq D \min _{i} \forall i \in$ NPI.

Furthermore, the optimization model use a candidate diameter for each pipe based on a set of commercial diameters, Eq. (8) and the assignment of only one commercial diameter for each pipe, Eq. (9).

$D_{i}=\sum_{d=1}^{\mathrm{ND}} Y D_{d, i} \cdot D \operatorname{com}_{d, i} \forall i \in \mathrm{NPI}$,

$\sum_{d=1}^{\mathrm{ND}} Y D_{d, i}=1 \forall i \in \mathrm{NPI}$.

Where: NPI - number of pipes in the network; $C$ pipe $_{i}\left(D_{i}\right)$ - unit cost of pipe $i$ as a function of its diameter $D_{i} ; D_{i}-$ diameter of pipe $i ; L_{i}-$ length of pipe $i$; NPU - number of pumping stations in the network; NS - number of scenarios; $\operatorname{prob}_{s}$ - probability of scenario $s$; Cpenp - penalty coefficient for minimum pressure violations; $\mathrm{NN}$ - number of nodes;

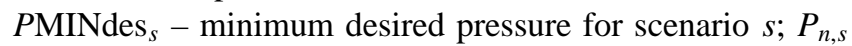
- pressure in node $n$ for scenario $s$; Cpend - penalty coefficient for demand violations; $Q D_{n, s}$ - demand in node $n$ for scenario $s ; Q C_{n, s}$ - consumption in node $n$ for scenario $s$; $Q \mathrm{ps}_{j}$ - highest pump discharge $\left(1 \mathrm{~s}^{-1}\right)$ for all the scenarios in PS $j ; H \mathrm{ps}_{j}$ - pumping head (m) for the highest discharge in PS $j ; \mathbf{I}_{n, i}$ - incidence matrix of the network; $Q_{i, s}$ - flow on the pipe $i$ in scenario $s ; \Delta H_{i, s}$ - head loss in pipe $i$ in scenario $s ; K_{i}, \alpha$-coefficients that depends of the physic characteristics of the pipe $i ; \mathrm{MAX}_{n, s}$ - maximum pressure in node $n$ for scenario $s$; $P \mathrm{MINadm}_{n, s}-$ minimum admissible pressure in node $n$ for scenario $s ; D \min _{i}-\operatorname{minimum}_{\text {diameter for }}$ the pipe $i$; ND - number of commercial diameters; $D \operatorname{com}_{d, i}$ - commercial diameter $d$ assigned to pipe $i ; Y D_{d, i}$ - binary variable to represent the use of the diameter $d$ in pipe $i$.

Two kinds of minimum pressure were considered in the model: the pressures can be lower than the desired pressure but not lower than the admissible pressure. If the nodal pressure values remain between these two limits the objective function is penalized. In addition, if the pressure is lower than the desired pressure the nodal demands will not be totally satisfied and the objective function is penalized as a function of the difference between the actual water demand and the demand that is satisfied (Cunha and Sousa, 2010). For pressure equal to or higher than the desired pressure the demand is totally satisfied and for pressures lower than the admissible pressure there is no nodal consumption.

\section{Case studies}

The model is applied to two similar case studies based on the network in Xu and Goulter (1999). In case study 1 (CS1),
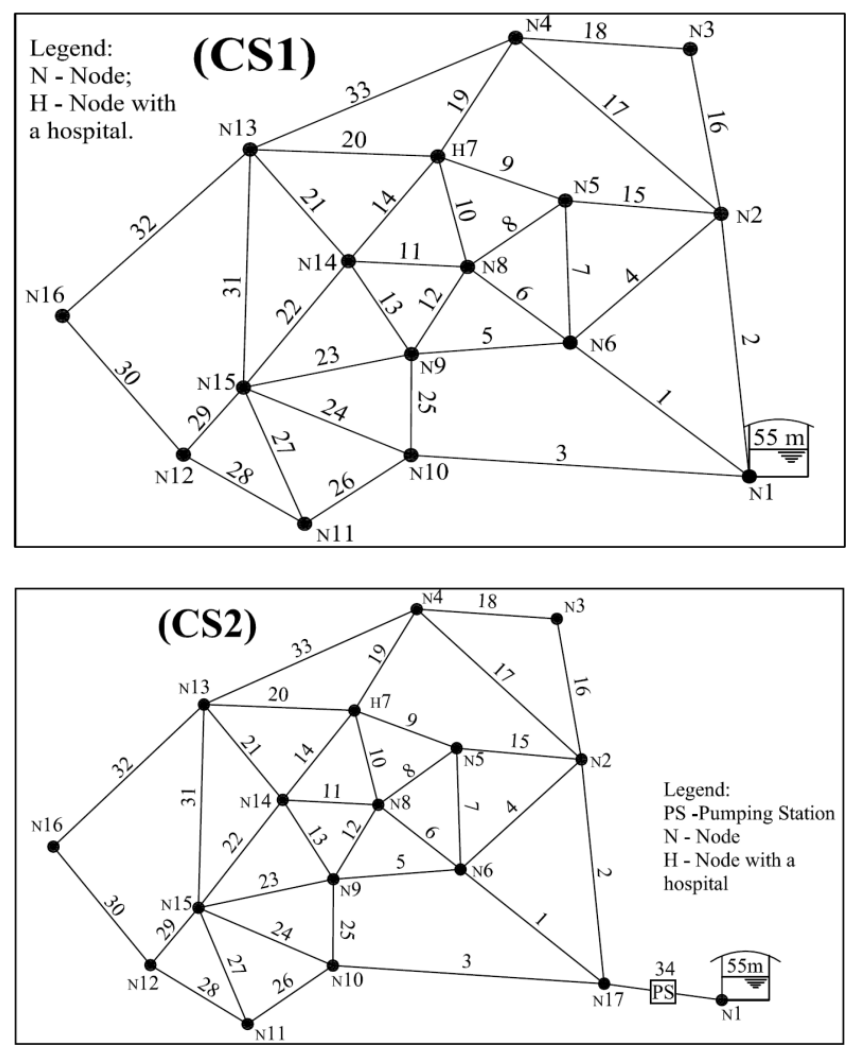

Figure 1. Network schemes: case study 1 (CS1) and case study 2 (CS2).

Fig. 1, the network is gravity fed by a single reservoir with a fixed level of $55 \mathrm{~m}$ and comprises 33 pipes and 16 nodes. Case study 2 (CS2), Fig. 1, is similar but it introduces a PS downstream of the reservoir (link 34). This PS is a contingency structure that should be used only in abnormal working conditions. As these situations are usually short-lived, the energy consumption and its cost were neglected.

The characteristics of the pipes are given in Table 1 and the nodes in Table 2. The commercial diameters (and their cost) used in the present study are given in Table 3. The head losses were calculated using the Hazen-Williams equation. It is also assumed that there is a hospital in node 7 with special pressure and demand requirements.

A multiple scenario approach was used to design the network for the two case studies:

- Scenario 1: Instantaneous peak discharge (IPD);

- Scenario 2: IPD and pipe 1 out of service;

- Scenario 3: IPD and pipe 2 out of service;

- Scenario 4: IPD and pipe 3 out of service;

- Scenario 5: IPD and a fire in node $3\left(2001 \mathrm{~s}^{-1}\right)$; 
Table 1. Characteristics of the pipes.

\begin{tabular}{cccccccc}
\hline Pipe & $\begin{array}{c}\text { Initial } \\
\text { node }\end{array}$ & $\begin{array}{c}\text { Final } \\
\text { node }\end{array}$ & $\begin{array}{c}\text { Length } \\
(\mathrm{m})\end{array}$ & Pipe & $\begin{array}{c}\text { Initial } \\
\text { node }\end{array}$ & $\begin{array}{c}\text { Final } \\
\text { node }\end{array}$ & $\begin{array}{c}\text { Length } \\
(\mathrm{m})\end{array}$ \\
\hline 1 & 1 & 6 & 3660 & 18 & 3 & 4 & 1830 \\
2 & 1 & 2 & 3660 & 19 & 7 & 4 & 1830 \\
3 & 1 & 10 & 3660 & 20 & 7 & 13 & 1830 \\
4 & 6 & 2 & 2740 & 21 & 14 & 13 & 1830 \\
5 & 6 & 9 & 1830 & 22 & 14 & 15 & 1830 \\
6 & 6 & 8 & 1830 & 23 & 9 & 15 & 1830 \\
7 & 6 & 5 & 1830 & 24 & 10 & 15 & 1830 \\
8 & 5 & 8 & 1830 & 25 & 9 & 10 & 1830 \\
9 & 5 & 7 & 1830 & 26 & 10 & 11 & 1830 \\
10 & 8 & 7 & 1830 & 27 & 11 & 15 & 2740 \\
11 & 8 & 14 & 1830 & 28 & 11 & 12 & 1830 \\
12 & 8 & 9 & 1830 & 29 & 12 & 15 & 1830 \\
13 & 9 & 14 & 1830 & 30 & 12 & 16 & 1830 \\
14 & 14 & 7 & 1830 & 31 & 15 & 13 & 1830 \\
15 & 2 & 5 & 1830 & 32 & 16 & 13 & 3660 \\
16 & 2 & 3 & 1830 & 33 & 13 & 4 & 3660 \\
17 & 2 & 4 & 2740 & 34 & 1 & 17 & Pump \\
\hline
\end{tabular}

- Scenario 6: IPD and a fire in node $12\left(2001 \mathrm{~s}^{-1}\right)$;

- Scenario 7: IPD and a fire in node $13\left(2001 \mathrm{~s}^{-1}\right)$.

The IPD is 1.8 times the average discharge. For case study 2 , the maximum nodal pressures should not exceed $60 \mathrm{~m}$ for scenario 1 and should not exceed $90 \mathrm{~m}$ for scenarios 2 to 7 , for the nodes of the network (N2 to N16). In the pipe break scenarios (2 to 4), it is assumed that the pipe that breaks can be isolated without compromising the supply of the respective end nodes. For scenario 1, the minimum desired and admissible pressures are $30 \mathrm{~m}$ for all nodes; for scenarios 2 to 7 the minimum desired pressure is $25 \mathrm{~m}$ and the minimum admissible pressure is $10 \mathrm{~m}$ for all nodes except node 7 ; as node 7 supplies a hospital, for scenarios 2 to 7 the minimum desired pressure is $30 \mathrm{~m}$ and the minimum admissible pressure is $25 \mathrm{~m}$. In scenarios 5 to 7 it is assumed that the firefighting demands are completely satisfied even if the fire node pressure is lower than the desired pressure.

\section{Results and comparisons}

This work proposes a different approach to toughening a WSS so that it can cope with normal and abnormal situations and then compares it with another possible solution. In both case studies the network must work under 7 different operating scenarios (the traditional peak design flow and 6 extreme scenarios -3 burst pipe scenarios and 3 firefighting scenarios). The objective function of the robust optimization model includes pipe costs, pumping station costs (construction and equipment) and penalties for pressure and demand violations. Network robustness can only be achieved in case study 1 by increments in pipe diameters. The flow must have alternative paths with enough capacity to carry bigger discharges to overcome the extreme scenarios. Network robust-
Table 2. Characteristics of the nodes.

\begin{tabular}{cccccc}
\hline Node & $\begin{array}{c}\text { Ground } \\
\text { Elevation } \\
(\mathrm{m})\end{array}$ & $\begin{array}{c}\text { Peak } \\
\text { Discharge } \\
\left(\mathrm{l} \mathrm{s}^{-1}\right)\end{array}$ & $\begin{array}{c}\text { Node } \\
\begin{array}{c}\text { Ground } \\
\text { Elevation } \\
(\mathrm{m})\end{array}\end{array}$ & $\begin{array}{c}\text { Peak } \\
\text { Discharge } \\
\left(\mathrm{l} \mathrm{s}^{-1}\right)\end{array}$ \\
\hline 1 & 0 & 0 & 10 & 0 & 43.889 \\
2 & 0 & 43.889 & 11 & 0 & 43.889 \\
3 & 0 & 43.889 & 12 & 0 & 43.889 \\
4 & 0 & 43.889 & 13 & 0 & 43.889 \\
5 & 0 & 43.889 & 14 & 0 & 43.889 \\
6 & 0 & 43.889 & 15 & 0 & 43.889 \\
7 & 0 & 43.889 & 16 & 0 & 43.889 \\
8 & 0 & 43.889 & 17 & 0 & 0 \\
9 & 0 & 43.889 & & & \\
\hline
\end{tabular}

ness can also be achieved in case study 2 by using the pumping station to increase the head at the reservoir. For the extreme scenarios, which occur occasionally and only for short periods of time, it was assumed that the maximum nodal pressure should not exceed $90 \mathrm{~m}$ (this constraint limits the pumping head and avoids potentially excessive pressure in the network). This approach avoids the large pipe diameter increase imposed by the case study 1 conditions (gravity fed network).

The decision variables of the robust optimization model are: case study 1 - pipe diameters; case study 2 - pipe diameters and pumping head for scenarios (2 to 7) of fixed velocity pumps. The peak discharge design (PDD) is determined by solving the model considering only scenario 1 . This design is used to compare the cost differences that the robustness solutions imply. To synthesize the results, only the PDD solution, the low robustness design (LRD) and the high robustness design (HRD) for each of the two case studies are presented. However, intermediate robust solutions can be achieved by considering different levels of robustness for the network (Cunha and Sousa, 2009). The LRD assumes a low probability of the extreme scenarios occurring and includes small penalty coefficients. The HRD is obtained assuming a high probability that the extreme scenarios will occur and large penalty coefficients. Figures 2 and 3 show the details of the solutions found for case studies 1 and 2. These figures show the commercial diameter chosen for each pipe in millimetres, the PS head in meters for the different scenarios considered, the partial and total cost of the solutions and also the total pressure and demand violations.

The "Total pressure violations" given in Figs. 2 and 3 represent the sum of all the pressure violations at all the network nodes and for all the scenarios. A similar procedure was used to compute the "Total demand violations".

The figures show that pressure and demand violations are reduced by enlarging some pipes and the pumping heads, meaning that more reliable solutions imply higher costs. The HRD presented illustrates that the robust design enlarges the pipe diameters by creating "main rings", which provide extra redundancies to supply all the nodes - even for the extreme 
Table 3. Commercial diameters, unit costs and Hazen-Williams coefficients.

\begin{tabular}{cccccc}
\hline $\begin{array}{c}\text { Diameters } \\
(\mathrm{mm})\end{array}$ & $\begin{array}{c}\text { Unit cost } \\
(€ / \mathrm{m})\end{array}$ & $\begin{array}{c}\text { H-W } \\
\text { Coefficients }\end{array}$ & $\begin{array}{c}\text { Diameters } \\
(\mathrm{mm})\end{array}$ & $\begin{array}{c}\text { Unit cost } \\
(€ / \mathrm{m})\end{array}$ & $\begin{array}{c}\text { H-W } \\
\text { Coefficients }\end{array}$ \\
\hline 100 & 87 & 120 & 450 & 247 & 120 \\
125 & 97 & 120 & 500 & 277 & 120 \\
150 & 102 & 120 & 600 & 371 & 120 \\
200 & 120 & 120 & 700 & 465 & 120 \\
250 & 147 & 120 & 800 & 559 & 120 \\
300 & 157 & 120 & 900 & 653 & 120 \\
350 & 187 & 120 & 1000 & 747 & 120 \\
400 & 215 & 120 & & & \\
\hline
\end{tabular}

Table 4. Total cost differences for the two case studies.

\begin{tabular}{llll}
\hline & $\begin{array}{l}\text { Peak } \\
\text { Discharge } \\
\text { Design (PDD) }\end{array}$ & $\begin{array}{l}\text { Low } \\
\text { Robustness } \\
\text { Design (LRD) }\end{array}$ & $\begin{array}{l}\text { High } \\
\text { Robustness } \\
\text { Design (HRD) }\end{array}$ \\
\hline & Case study 1 & \\
\hline Total cost $=$ Pipe costs $€\left(\times 10^{6}\right)$ & 9.890 & 10.557 & 11.975 \\
Pressure violations $(\mathrm{m})$ & 0 & 218.64 & 1.87 \\
Demand violations $\left(1 \mathrm{~s}^{-1}\right)$ & 0 & 341.71 & 1.62 \\
\hline Difference in total costs & $0 \%$ & $+7 \%$ & $+21 \%$ \\
\hline & Case study 2 & & \\
\hline Total cost $€\left(\times 10^{6}\right)$ & 9.890 & 10.997 & 11.397 \\
Pipe costs $€\left(\times 10^{6}\right)$ & 9.890 & 10.337 & 10.442 \\
Pump costs $€\left(\times 10^{6}\right)$ & 0 & 0.659 & 0.975 \\
Pressure violations $(\mathrm{m})$ & 0 & 230.53 & 0.25 \\
Demand violations $\left(1 \mathrm{~s}^{-1}\right)$ & 0 & 363.48 & 0.36 \\
\hline Difference in total costs & $0 \%$ & $+11 \%$ & $+15 \%$ \\
\hline
\end{tabular}

scenarios considered. It should also be pointed out that those "main rings" always embrace the critical node - Hospital (H7). As expected, the case study 1 solutions use larger pipe diameters than case study 2. In fact, the PS plays an important role in ensuring the network supply for case study 2, instead of using larger pipe diameters; reliability is achieved by the PS increasing the head at the reservoir for the extreme scenarios.

Table 4 shows a comparison of the solutions obtained for the case studies (cost, pressure violations and demand violations, for the designs presented in Figs. 2 and 3). The increases in total costs for the LRD and the HRD are calculated taking the PDD cost as reference. The penalty coefficients for the two case studies were fixed so as to obtain solutions with similar pressure and demand violations for both case studies.

Some conclusions can be drawn from Table 4. In case study 1 , the LRD costs are $7 \%$ higher, but to get an HRD would require spending $21 \%$ more than the cost of the traditional PDD solution. As robustness is achieved solely by enlarging the pipe diameters, the HRD for case study 1 has the highest total cost for the pipes $-11.975 \times 10^{6} €$ (this is the design with largest pipe diameters). In terms of network behaviour, this design is sufficiently reliable to perform well even in the extreme scenarios. However, for normal working conditions the pipes are overdesigned, which means low velocities and high residence times, conditions that may lower water quality and safety. The option to raise the reliability of a WSS to high levels only by increasing the pipe diameters should therefore be avoided if there are other alternatives that can be implemented.

The LRD for case study 2 is more costly than that for case study 1 . These case studies show that, in terms of cost, for low robustness designs it is preferable to enlarge the pipes instead of using a PS. For less reliable solutions, a minor increase of pipe diameters is required for the network which will be cheaper than implanting a pumping station downstream of the reservoir, even for low pumping heads.

Finally, the cost of the HRD for case study 2 is $15 \%$ higher than the PDD solution cost. This design is achieved both by increasing the pipe diameters and by using the PS to cope 

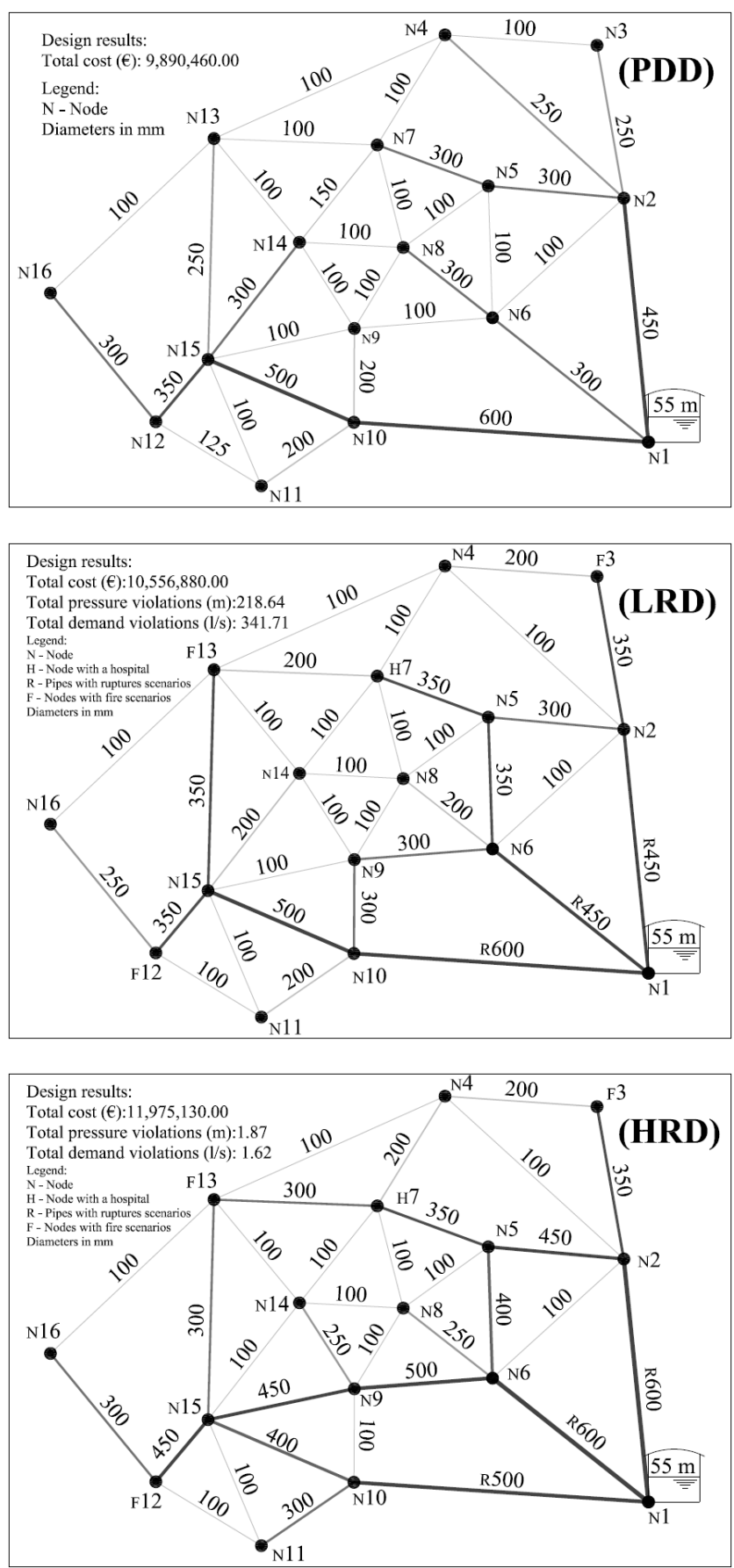

Figure 2. Designs for case study 1: (PDD) peak discharge (LRD) low robustness and (HRD) high robustness.

with the extreme scenarios. The combination of these elements resulted in a high robustness design for a lower cost increase than case study 1 . Furthermore, this approach reduces the overdesign problems. By introducing additional power at the reservoir, the PS avoids enlarge pipes to ensure the minimum desired pressures at the network nodes. In conclusion, these case studies indicate that for high robustness designs it
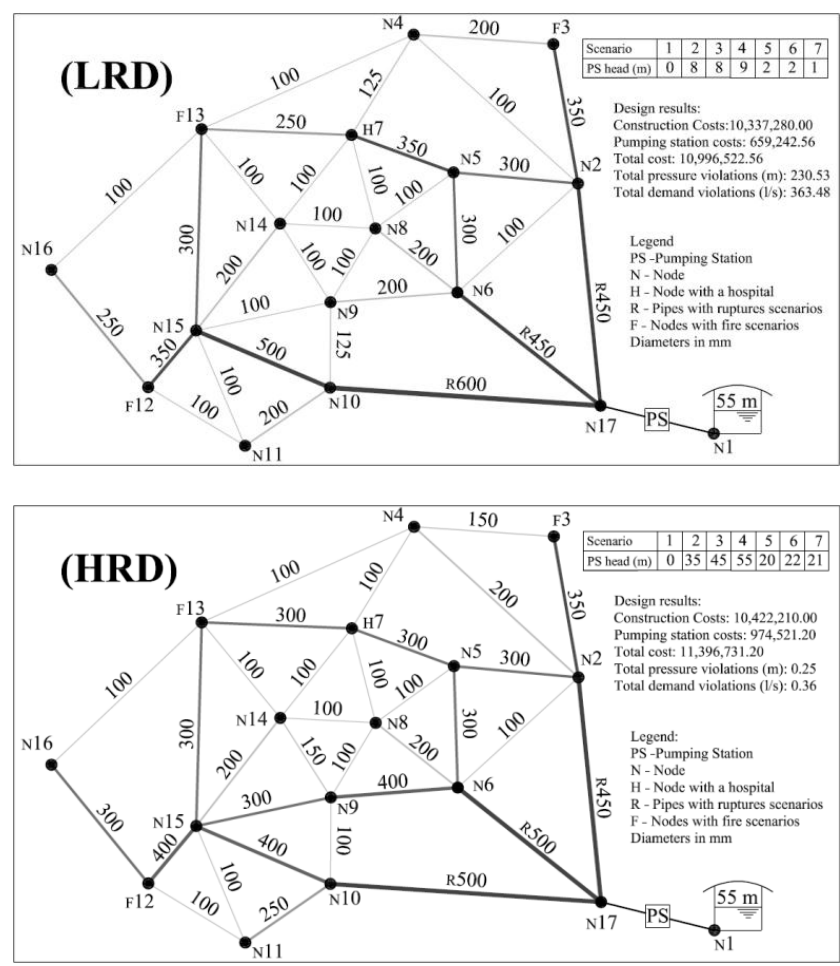

Figure 3. Designs for case study 2: (LRD) low robustness and (HRD) high robustness.

is preferable to use a PS combined with smaller enlarging of the pipes than to rely on more general of the pipes.

\section{Conclusions}

To obtain high robustness solutions WSSs must be designed to cope with extreme operating conditions during their life cycle. The uncertainty related to future operating conditions should be taken into account early in the design stage. This work has presented a robust optimization model to help decision makers attain a good trade-off between reliability and cost. The performance of this method was illustrated by means of two case studies. The reliability of the water supply systems was ensured by two different strategies: 1st - designing the system to cope with the extreme operating conditions by increasing the pipe diameters; 2 nd - designing the system for normal operating conditions and introducing a pumping station to deal with the extreme operating conditions.

This approach provides a new technique to toughen up a WSS and also compares, in terms of costs, the solutions arrived at by different ways. The case studies used to test the model led to the following conclusions: for low robustness solutions the 1st strategy was less expensive; if a high robustness solution is required then the 2nd strategy is less expensive. It must be also pointed out that the 1st strategy overdesigns the pipe diameters, leading to low velocities and 
high water residence times. The 2 nd strategy, which is innovation proposed in this work, can also be viewed as an alternative for existing WSSs. For some existing systems, strengthening the infrastructure links may be difficult if it involves construction works in urban areas and it could also be prohibitively expensive, so innovative strategies should be used. For future developments of this work, consideration of the water age can be added to the determination of solutions. The water quality could be used to evaluate the design alternatives so that the solution can be further optimized for a truly robust design. It could also be important to understand the influence of the maintenance costs of many pumping stations required as contingence infrastructures in large systems, which is likely the case in real water systems. A life cycle cost analysis of the strategies (including the maintenance of pipes and pumps) can be conducted to choose the design of a robust solution.

Acknowledgements. This work has been financed by FEDER funds through the Programa Operacional Factores de Competitividade - COMPETE, and by national funds from FCT - Fundação para a Ciência e Tecnologia under grant PTDC/ECM/64821/2006. The participation of the first author in the study is supported by FCT - Fundação para a Ciência e Tecnologia through Grant SFRH/BD/47602/2008.

Edited by: R. Farmani

\section{References}

Aarts, E. and Korst, J.: Simulated Annealing and Boltzmann Machines: A Stochastic Approach to Combinatorial Optimization and Neural Computing, Chichester, England, John Wiley \& Sons, 1989.
Babayan, A. V., Savic, D. A., Walters, G. A., and Kapelan, Z. S.: Robust Least-Cost Design of Water Distribution Networks Using Redundancy and Integration-Based Methodologies, J. Water Res. Pl.-ASCE, 133, 67-77, 2007.

Carr, R. D., Greenberg, H. J., Hart, W. E., Konjevod, G., Lauer, E., Lin, H., Morrison, T., and Phillips, C. A.: Robust optimization of contaminant sensor placement for community water systems, Math. Program., 107, 337-356, 2006.

Cunha, M. C. and Sousa, J.: Water Distribution Network Design Optimization: Simulated Annealing Approach, J. Water Res. Pl.ASCE, 125, 215-221, 1999.

Cunha, M. C. and Sousa, J.: Hydraulic Infrastructures Design Using Simulated Annealing, J. Infrastruct. Syst., 7, 32-39, 2001.

Cunha, M. C. and Sousa, J.: Robust design of water distribution networks: a comparison of two different approaches, in: Proc. CCWI, Sheffield, UK, CRC Press, 181-187, 2009.

Cunha, M. C. and Sousa, J. J. O.: Robust Design of Water Distribution Networks for a Proactive Risk Management, J. Water Res. Pl.-ASCE, 136, 227-236, 2010.

DIEDE and AIDIS: Integrated risk management to protect drinking water and sanitation services facing natural disasters, IRC International Water and Sanitation Centre, The Netherlands, thematic overview paper no 21, 54 pp., 2008.

Giustolisi, O., Laucelli, D., and Colombo, A. F.: Deterministic versus Stochastic Design of Water Distribution Networks, J. Water Res. Pl.-ASCE, 135, 117-127, 2009.

Jeong, H. S., Qiao, J., Abraham, D. M., Lawley, M., Richard, J., and Yih, Y.: Minimizing the Consequences of Intentional Attack on Water Infrastructure, Comput.-Aided Civ. Inf., 21, 79-92, 2006.

Mulvey, J., Vanderbei, R., and Zenios, S.: Robust Optimization of Large-Scale Systems, Oper. Res., 43, 264-281, 1995.

$\mathrm{Xu}, \mathrm{C}$. and Goulter, I. C.: Reliability-Based Optimal Design of Water Distribution Networks, J. Water Res. Pl.-ASCE, 125, 352362, 1999. 